\title{
EFFECTS OF CONSISTENCY VS. VARIABILITY IN ROBOTICALLY CONTROLLED TRAINING OF STEPPING IN ADULT SPINAL MICE
}

\author{
L.L. Cai ${ }^{1}$; A.J. Fong ${ }^{1}$; C.K. Otoshi ${ }^{3}$; Y.Q. Liang ${ }^{2}$; J.G. Cham²; H. Zhong ${ }^{4}$; R.R. Roy ${ }^{5}$ V.R. Edgerton ${ }^{3,4,5}$; J.W. \\ Burdick $^{1,2}$; \\ 1. Bioengineering, ${ }^{2 .}$ Mechanical Engineering, California Institute of Technology, Pasadena, CA, USA \\ ${ }^{3 .}$ Neurobiology, ${ }^{4}$ Physiological Science, ${ }^{5 .}$ Brain Research Institute, UCLA, Los Angeles, CA, USA
}

\begin{abstract}
This paper studies the possible benefit that can be obtained by introducing variability into the robotic control of trajectories used to train hindlimb locomotion in adult spinal mice. The spinal cords of adult female SwissWebster mice were completely transected at a mid-thoracic level. Fourteen days post-transection, the spinal mice were robotically trained to step in the presence of a 5-HT agonist, quipazine, for a period of six weeks. In this pilot study nine animals were divided into three groups, each receiving a different control strategy: a fixed training trajectory (Group A), a variable training trajectory without interlimb coordination imposed (Group B) and a variable training trajectory with hindlimb bilateral coordination imposed (Group C). Preliminary results indicate that Group A recovers more slowly than the two groups receiving variable modes of robotic training. Groups $\mathrm{B}$ and $\mathrm{C}$ show higher levels of recovery than Group A in terms of the number of steps performed during testing sessions, as well as in their step periodicity and shape consistency. Group C displays a higher incidence of alternating stepping than Group B. These results indicate that variable trajectory robotic training paradigms may be more effective than fixed trajectory paradigms in promoting robust post-injury stepping behavior. Furthermore, it appears that the inclusion of interlimb coordination is an important contribution to successful training.
\end{abstract}

\section{INTRODUCTION}

Robotic-facilitated rehabilitative training paradigms have recently become more common. Commercially available robotic orthotics such as the Lokomat $\mathrm{TM}$ are already available to facilitate the rehabilitative training of spinal cord injuried (SCI) and stroke patients with promising results [1]. However, little is known about the mechanisms of how locomotor recovery is achieved with this type of rehabilitative training. Additionally the best training procedure has yet to be established.

To shed light on these issues, we have developed a robotic training device (Fig. 1) to study locomotor recovery after $\mathrm{SCI}$ in mice. A mouse model is attractive due to the many strands of transgenic mice available. In our previous experiments, we have demonstrated that fixed trajectory robotic training and serotonergic treatment each have significant independent and synergistic positive effects in enhancing motor performance of adult spinalized mice [2]. Furthermore, these improvements were retained even when both training and drug treatment were suspended, suggesting that a type of learning phenomenon had occurred [2]. In this paper, we propose the hypothesis that the post SCI, spinal cord will be better able to relearn to step if it is constantly challenged during locomotor training by introducing forms of variability in the training pattern.

The present pilot study compared the efficacy of two variable trajectory robotic training paradigms versus fixed trajectory robotic training on recovering locomotor ability in completely spinalized adult mice that were administered quipazine. The results of the study demonstrate that animals given variable robotic training exhibited faster and more pronounced recovery than those given fixed robotic training. These findings suggest that an optimal rehabilitation strategy can be obtained by combining an "assist as needed" training paradigm and pharmacological intervention.

\section{METHODS}

Animals: Adult Swiss-Webster mice (mean body weight of $21.5 \pm 1.1 \mathrm{~g}$ at the time of surgery) obtained from Charles River Laboratories (Wilmington, MA) were used. A complete mid thoracic spinal transection was performed at 45 days of age.

Quipazine Administration: A serotonergic agonist quipazine $(0.5 \mu \mathrm{g} / \mathrm{g}$ body weight, i.p. $)$ was administered to all subjects $5 \mathrm{~min}$ prior to the training or testing session [3].

Robotic Step-Training and Measurement System: A four axis robotic system was developed for position data collection and as an active training apparatus. The robotic system consists of four major components: 1) a pair of robotic manipulators to guide ankle motion; 2) motion control hardware; 3) a treadmill; and 4) a body weightsupport device. The main components of the mouse stepper are the robotic manipulators. Each robotic manipulator is comprised of a leg guidance linkage (Fig. 2), a pair of motors that drive the linkage (2342-006CR; Micromo Electronics, Clearwater, FL), and a pair of optical encoders that record the rotational position of the motors (Agilent HEDM-5500; Micromo Electronics). Ankle position is derived from these encoders and the linkage 


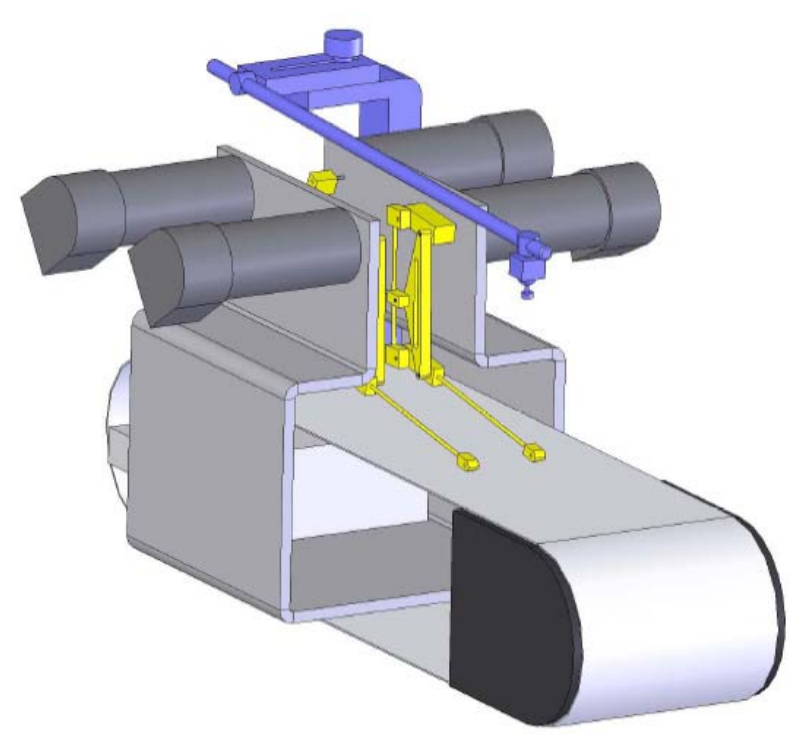

Figure 1: Schematic of Current Step Training System

geometry. The five bar limb guidance linkage allows optimal motion sensing and control in a $3.5 \times 3.5 \mathrm{~cm}$ workspace, which is sufficient to accommodate all step trajectories associated with mouse locomotion. In addition, due to the geometry of the linkage, the dynamic of the two axes are uncoupled and approximately linear in the range of motions encountered during training and evaluation.

The mice are placed into the mouse stepper using a coneshaped cloth harness. Once the mouse has entered the harness, it is secured using a binder clip, which is then magnetically attached to the weight-support system.

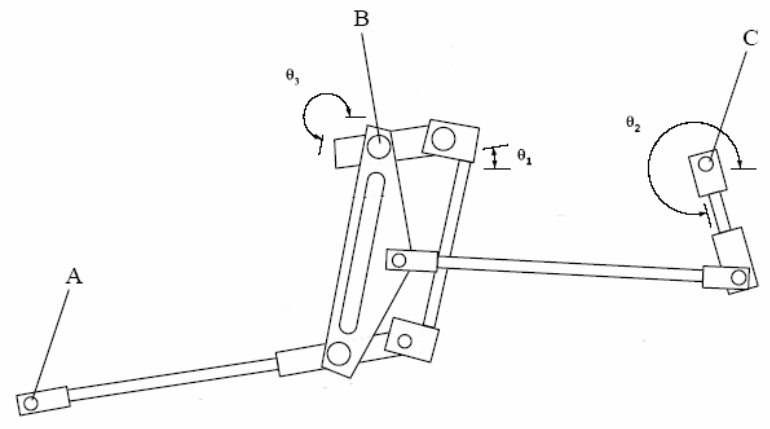

Figure 2: Schematics of the five-bar, parallelogramtype linkage used. The dimension and weight of each link is noted. The drawing is done at actual scale. Important features labeled: A) ankle attachment point; B) motor attachment point driving linkage 4; C) motor attachment point driving linkage 1 .

Fixed Trajectory Robotic Training: In fixed robotic training, the robotic arms are controlled using a PID controller to perform a continuous trajectory tracking of a pre-determined pattern. When attach to the mice, the robotic arms actively move the ankle points of the limbs in this fixed trajectory. The imposed $\mathrm{x}$ and $\mathrm{y}$ trajectory of each hindlimb was obtained from a neonatally transected mouse that stepped well. This pattern was recorded from the neonatal mouse when it was approximately the same age as the adult mice used in this study. Neonatally transected mice can spontaneously recover functional stepping without pharmacological or mechanical assistance (unpublished observations). The spinal mice were trained for 10 min. a day, 5 days per week.

Robotic Training with Variability I (Band) Algorithm: The animals in this group received a variable training paradigm that provided an "assist as needed" training strategy. This training paradigm uses velocity fields to control the movement of the robotic linkages. That is, the velocity of the robot's distal tip is commanded to a specific speed. This strategy implements two fixed boundaries: an inner bound and an outer bound, forming a "band" surrounding the desired trajectory. Within the band, the robot follows a small velocity field tangent to the desired trajectory. I.e., it nudges the leg along the trajectory, but does not enforce specific timing of leg movement. The regions inside (outside) the band are filled with an outward spiraling (inward-spiraling) converging velocity field. When the ankle point of the hindlimb leaves the band, the convergent velocity fields will rapidly move it back into the band region (Fig. 3). However, the paradigm does not impose interlimb constraint. In essence the robotic device only actively manipulates the hindlimbs when their position deviates from the nominal trajectory beyond the predetermined boundaries. Inside the band, the animal largely dictates its own motions, with a small forward bias provided by the robot. The animals were trained for 10 min. each day, 5 days a week.

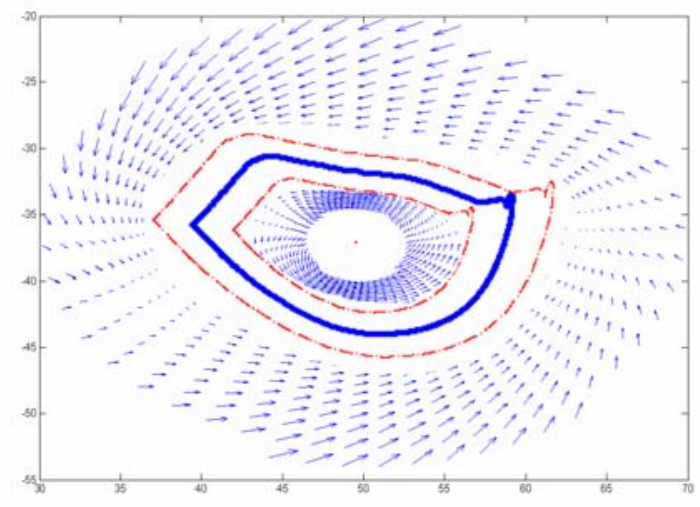

Figure 3: Variability training paradigm I (Band). The desired training trajectory is shown in thick blue. The dotted thin red lines represent the boundaries. The blue arrows correspond to the convergent velocity fields.

Robotic Training with Variability II (Window) Algorithm: The third group received a variable training paradigm analogous to the second group, but based on a "window" variable training paradigm. In this approach, a circular "window" moves along the desired trajectory. (Fig. 4) Within the window, a small velocity field tangent to the desired trajectory biases the robot's motion, but doesn't enforce specific timing inside window. Outside the 
boundary, the robotic movement is guided by a radial force field that points inward with a magnitude proportional to the distance from the circle center. Hence, when the animal's ankle deviates from the window, it is quickly returned to the window. Within the window, the ankle is gently guided in the direction of the trajectory, thus providing loose timing control. The same strategy was used on both hindlimbs and was timed to provide alternating phasing. The animals were trained for $10 \mathrm{~min}$ each day, 5 days a week.

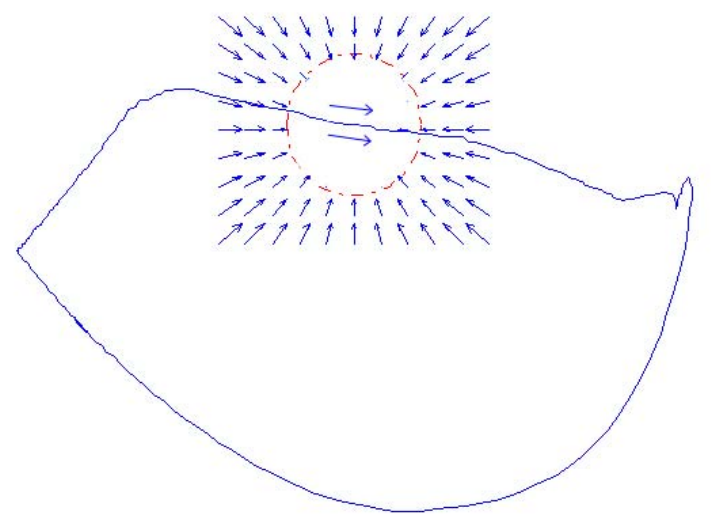

Figure 4: Variability training paradigm II (Window). The desired training trajectory is in blue and the moving window is highlighted in red. The blue arrows correspond to the radial force fields.

Experimental Design: Nine mice were randomly divided into three groups. All three groups received a form of robotic training with quipazine for six weeks. The first group was the control in this experiment and was trained with the fixed robotic training algorithm. The two experimental groups were each trained with a form of variable training. The second group received the "Band" variable training, while the third group received the "window" variable training for six weeks.

Data Acquisition and Formatting: Three modes of data were collected for each test session. First, using in-house acquisition programs written in the LabVIEW $^{\mathrm{TM}}$ development environment, we recorded position data for both ankles at $400 \mathrm{~Hz}$. Second, we maintained a log of all qualitative observations in a database. Third, beginning with the third test session, we captured video footage of both the left and right sides of each mouse as it stepped on the treadmill.

Data Analysis: We used Fast Fourier Transform (FFT) analysis to quantify step periodicity. Mice that stepped rhythmically exhibited a sharp and distinct fundamental peak in the FFT of their ankle trajectories. The location of the predominant peak along the $\mathrm{x}$-axis corresponds to the most common stepping frequency. Conversely, mice with poor periodicity exhibited either a very broad fundamental peak in the FFT spectrum or, in extreme cases, failed to demonstrate a fundamental peak. To quantify these observations, we measured the full width at half maximum (FWHM) of the fundamental peak. A low measurement of FWHM corresponds to temporally consistent, rhythmic stepping, whereas high measurements typically indicate erratic stepping consistent with stumbling and foot dragging.

In addition to the temporal information provided by FFT analysis, we also sought to study any changes in spatial stepping consistency throughout the study. We suggest that locomotor improvement can be characterized by more consistent repetition of a nominal trajectory. Principle components analysis (PCA) is a multivariate analysis technique that 1) picks out patterns (relationships) in the variables of a data set; and 2) reduces the dimensionality of the data set without significant loss of information [4]. Using PCA, we can calculate the percentage of the total variance in the data set that is captured by the first principal component. Hence, a high PCA score corresponds to a subject that consistently executes the same stepping trajectory.

\section{RESULTS}

The average total number of steps performed in a best 15 second interval by the animals in each group is shown in figure 5. The "window" and "band" groups performed more steps than the fixed group. However, there does not seem to be any statistical difference between the two variable groups.

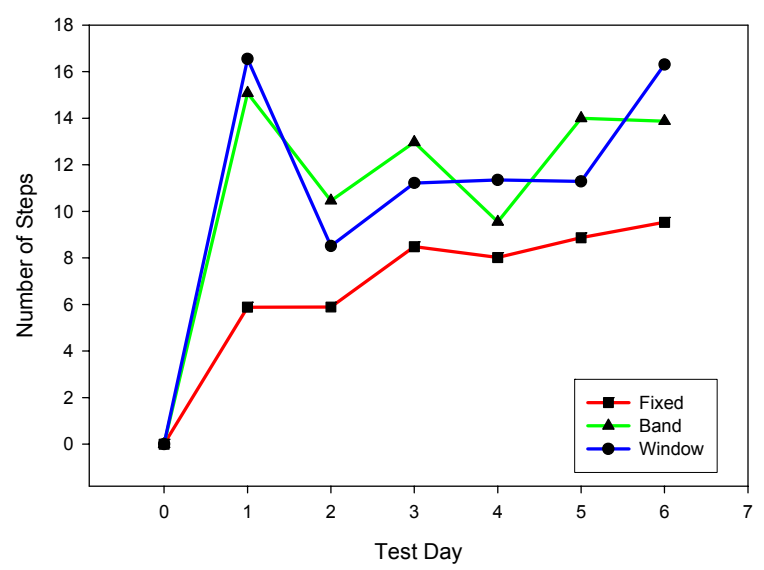

Figure 5: Number of steps performed in $15 \mathrm{sec}$. interval. Through out the experiment, both variable training groups perform better compare to the fixed group.

In figure 6, the inverse of FWHM is plotted to emphasize the improvement in step rhythm throughout the study. The "window" and "band" variable training groups consistently stepped more rhythmically than the fixed training group. Although the two variable groups could not be statistically distinguished, the "window" group seemed to recover faster. A 0 score was assigned to all mice that could not step on test day 0 . 
PCA percentage is plotted in figure 7. Although the "window" training group appeared to recover faster, there was no indication of a difference between the step shape consistencies of the three groups. This may be due to the small $\mathrm{n}$ used. Alternatively, this supports previous findings that quipazine, rather than the specific training pattern, is primarily responsible for shape consistency [2]. A PCA percentage of $45 \%$ assigned to for all mice that could not step on test day 0 .

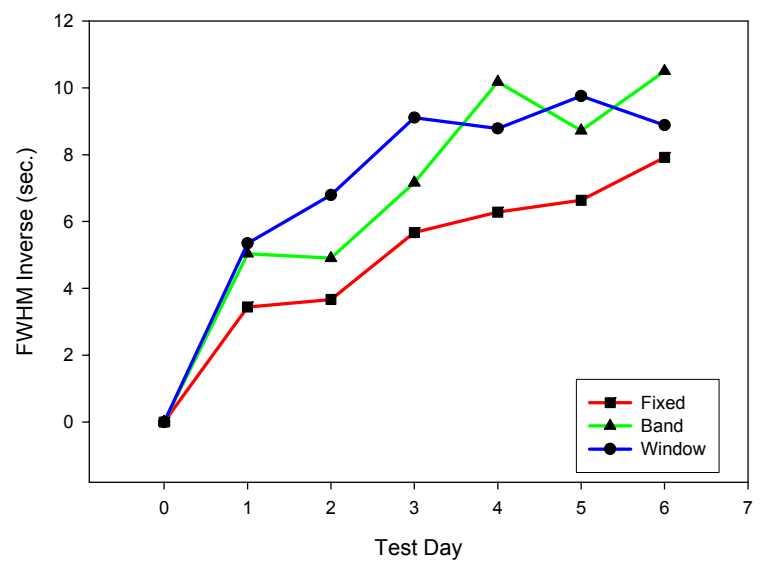

Figure 6: The inverse of FWHM is plotted to emphasize the locomotor improvement over time. Both variable training groups performed better than the fixed training through out the experiment.

\section{DISCUSSION}

A key objective of this study was to ascertain whether providing variability in training enhances stepping recovery. A significant concern is that fixed trajectory training strategies may drive the spinal cord into a state of "learned helplessness," [5, 6] whereby the spinal cord, not challenged to explore potential stepping patterns on its own, will defer to the trainer and give up on learning. No statistically significant differences were observed between the variable "band" and "window" training groups. This may be due to the small $\mathrm{n}$ used. Nevertheless, both variable training paradigms appears to be a more effective rehabilitative strategy then the fixed training paradigm.

Nevertheless, interlimb coordination must be controlled in order to maximize locomotor recovery [7]. We found that similar is true for adult spinal mice. Although mice trained with the "band" pattern recovered greatly in their stepping performance, their steps were typically arrhythmic and frequently interrupted by dragging. In contrast, bouts of stepping executed by the "fixed" and "window" training groups were rhythmic and prolonged. This is clearly demonstrated by the FFT data, which show that stepping in the latter two groups converged to a frequency near $1 \mathrm{~Hz}$ as the study progressed, a result consistent with constant speed treadmill locomotion at $3 \mathrm{~cm} / \mathrm{sec}$.
Principal components analysis did not elucidate a difference in the shape consistency of the three groups. By week 3, the average PCA score of all three groups was greater than $80 \%$, indicating that all of the mice could step rhythmically. This result supports our previous conclusion that quipazine rather than step training has the larger influence on step shape consistency [2].

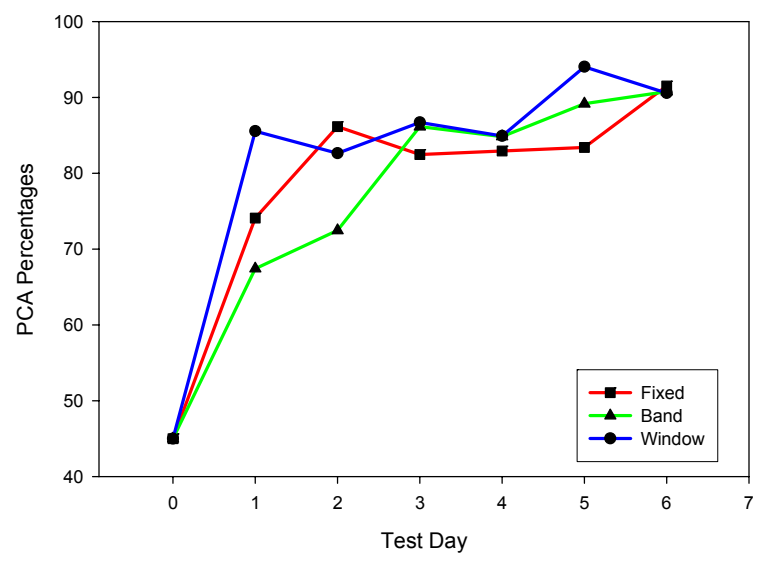

Figure 7: The PCA percentage score of each training gropu is plotted. There were no statistically significant differences between the three groups after the third week of training.

Sensory information is critical to motor learning $[8,9]$. The pattern and timing of assistance provided during step training dictates the extent to which the injured spinal cord can recover motor function. We have shown that failure to enforce contralateral phasing of the legs during training limits stepping recovery compared to mice trained with an alternating gait [2]. It is likely that the spinal cord is similarly highly sensitive to other proprioceptive cues. As demonstrated here, poorly conceived or poorly implemented training algorithms may actually inhibit stepping recovery.

This emphasizes a fundamental advantage of using robotic devices that can consistently and accurately apply defined locomotor training paradigms while simultaneously maintaining a thorough quantitative record of both training and performance parameters. Used with data optimization schemes, quantitative data are teaching us which parameters to focus on when devising novel training strategies. By developing our understanding of how sensory inputs affect spinal learning, we can "tune the spinal cord to learn."

\section{ACKNOWLEDGEMENT}

This work is supported by the Christopher Reeve Paralysis Foundation (\#VEC 2002-2) and the National Institutes of Health (1 R01 HD044830-01).

\section{REFERENCES}

[1] S. Hesse, H. Schmidt, C. Werner, and A. Bardeleben, "Upper and lower extremity robotic devices for rehabilitation and for studying motor control," Curr Opin Neurol, vol. 16, pp. 705-10, 2003. 
[2] A. J. Fong, L. L. Cai, C. K. Otashi, J. W. Burdick, and V. R. Edgerton, "EFFECTS OF QUIPAZINE AND ROBOTIC

TRAINING ON SPINAL MICE.," Proceedings of the 33rd Society

for Neuroscience Conference, New Orleans, LA, November 10, 2003.

[3] D. Feraboli-Lohnherr, J. Y. Barthe, and D. Orsal, "Serotonin-induced activation of the network for locomotion in adult spinal rats," $J$ Neurosci Res, vol. 55, pp. 87-98, 1999.

[4] G. H. Dunteman, Principal components analysis. Newbury Park, Calif: Sage Publications, 1989.

[5] J. W. Grau, D. G. Barstow, and R. L. Joynes, "Instrumental learning within the spinal cord: I. Behavioral properties," Behav Neurosci, vol. 112, pp. 1366-86, 1998

[6] R. N. Wool, D. Siegel, and P. R. Fine, "Task performance in spinal cord injury: effect of helplessness training," Arch Phys Med Rehabil, vol. 61 , pp. 321-5, 1980.

[7] S. F. Donker and P. J. Beek, "Interlimb coordination in prosthetic walking: effects of asymmetry and walking velocity," Acta Psychol (Amst), vol. 110, pp. 265-88, 2002.

[8] V. Dietz, R. Muller, and G. Colombo, "Locomotor activity in spinal man: significance of afferent input from joint and load receptors," Brain, vol. 125, pp. 2626-34, 2002.

[9] A. L. Behrman and S. J. Harkema, "Locomotor training after human spinal cord injury: a series of case studies," Phys Ther, vol. 80, pp. 688-700, 2000. 